\title{
Effects of MK-801 concentration on cell proliferation in rats with focal cerebral ischemia-reperfusion
}

\author{
Y.K. Gui ${ }^{1}$, L.L. Su', X.L. Niu', P. Zeng' ${ }^{1}$, R.R. Fang ${ }^{1}$, H.X. Lv² and P. Zhang ${ }^{1}$ \\ 1'Department of Neurology, the First Affiliated Hospital of Xinxiang Medical University, \\ Xinxiang, China \\ ${ }^{2}$ Department of Pharmacy, Xinxiang Medical University, Xinxiang, China \\ Corresponding author: P. Zhang \\ E-mail: pingzhangcn@126.com \\ Genet. Mol. Res. 14 (4): 12841-12847 (2015) \\ Received February 18, 2015 \\ Accepted May 18, 2015 \\ Published October 21, 2015 \\ DOI http://dx.doi.org/10.4238/2015.October.21.3
}

\begin{abstract}
We explored the relationship between MK-801 concentration and neural stem cell proliferation in rats with focal cerebral ischemiareperfusion $(F C I R)$. A total of 60 male Sprague Dawley rats were randomized into control (six rats), sham-operation (six rats), operation (12 rats), and MK- 801 groups. The MK- 801 group comprised 36 rats that were subjected to different doses of MK-801 $(0.2,0.4,0.6,0.8,1.0$, and 1.2 $\mathrm{mg} / \mathrm{kg}$ ). Suture occlusion was used to establish an ischemia reperfusion model of middle cerebral artery occlusion (MCAO); 30 min before establishing the FCIR model, the MK-801 group rats were intraperitoneally injected with different doses of MK-801, while the sham-operation and control groups were injected with normal saline. Seven days after model establishment, bromodeoxyuridine-positive cerebral cortex cells adjacent to the focus of infarction were labeled for immunohistochemistry. MK-801 at a concentration of $0.4 \mathrm{mg} / \mathrm{kg}$ prevented endogenous neural stem cell proliferation, and this inhibitory effect was strengthened with increasing MK-801 concentration, especially at concentrations greater than $0.8 \mathrm{mg} /$ $\mathrm{kg}$. MK-801 inhibits endogenous neural stem cell proliferation in rats with
\end{abstract}


FCIR, and the inhibitory effect is strengthened with increasing MK-801 concentration.

Key words: Focal cerebral ischemia reperfusion; FCIR; Neural stem cell; MK-801; Rat

\section{INTRODUCTION}

Neural stem cells (NSCs) are precursor cells that are capable of self-proliferation and multidirectional differentiation (Gage, 2000). Their proliferation and differentiation processes are considered a simplified model of nerve development and regeneration. Since Anderson and Michelsohn (1989) first put forward the concept of NSCs and experimentally confirmed their existence in 1989, exogenous and endogenous NSCs have been extensively investigated. Owing to low transplant survival rate and difficulty in differentiation control of exogenous NSCs, as well as ethical issues concerning NSC sources, endogenous NSCs have been the focus of cerebral infarction treatment. Research has demonstrated that N-methyl-D-aspartate (NMDA) receptor antagonists can reduce the area of cerebral infarction and protect neurons. Simultaneously, excitatory amino acids (EAAs) can activate endogenous NSCs through NMDA receptors, and promote their proliferation and differentiation to repair neurological function. Therefore, the control of NMDA receptor activation to achieve the best balance between neuron protection and activation of endogenous NSCs requires investigation. We performed intervention through intraperitoneal injection of NMDA receptor antagonist MK-801. We then observed nestin-positive cells in the cortex adjacent to the focus of infarction using immunohistochemistry (IHC), and explored the relationship between MK-801 concentration and activation of endogenous NSCs to provide a laboratory basis for clinical treatment and prevention of cerebral infarction.

\section{MATERIAL AND METHODS}

\section{Animals and grouping}

Sixty male Sprague Dawley rats weighing 250-300 g were randomized into nine groups as follows: 6 normal controls; 6 sham-operation controls; 12 operation controls; and 36 MK-801treated rats given different doses of MK-801 $(0.2,0.4,0.6,0.8,1.0$, and $1.2 \mathrm{mg} / \mathrm{kg})$. The MK-801 was provided by Shenzhen Jingmei Technology Co., Ltd. (Shenzhen, China). MK-801 at different concentrations was injected intraperitoneally into rats $30 \mathrm{~min}$ before operation. Rats were sacrificed on day 7 after operation. This study was carried out in strict accordance with the recommendations in the Guide for the Care and Use of Laboratory Animals of the National Institutes of Health. The animal use protocol was reviewed and approved by the Institutional Animal Care and Use Committee of Xinxiang Medical University.

\section{Establishment of experimental model}

Vascular occlusion using a nylon suture was performed in the rats to establish a rat model of middle cerebral artery occlusion (MCAO), according to the advanced protocol proposed by Longa et al. (1989). Rats were anesthetized by ip administration of $10 \%$ chloral hydrate (3 mL/ $\mathrm{kg}$ ), fixed in the supine position, and incised in the midline of the neck. The left common carotid 
artery (CCA), the internal carotid artery (ICA), and the external carotid artery (ECA) were exposed. The branches of the CCA and the ECA close to the heart were ligated with a nylon suture. A nylon suture was introduced into the ICA lumen through a micro-incision $5 \mathrm{~mm}$ from the branch of the CCA. The introduction was stopped after approximately $19 \mathrm{~mm}$ of nylon suture had been inserted and resistance felt, at which point the tip of the suture reached the origin of the middle cerebral artery (MCA) and caused occlusion. The MK-801 or the saline was ip injected immediately after the operation. After $2 \mathrm{~h}$, the rats were anesthetized with diethyl ether, all occlusion was removed, and $10 \mathrm{~mm}$ of nylon suture was withdrawn gently to restore blood flow. In the sham-operation controls, only $10 \mathrm{~mm}$ of nylon suture was inserted into the ICA lumen, but the rest of the procedure was the same as described above. A successful rat model includes indications of: 1) contralateral limb paralysis; 2) a positive result from the tail-hanging test; 3) reduced ipsilateral eye fissure; exclude non-standard rats; and 4) infarction and cerebral ischemic injury.

\section{TTC staining}

The rats from each group were anesthetized and sacrificed by decapitation $24 \mathrm{~h}$ after ischemia-reperfusion; the brains were instantly harvested and frozen. After 20 min, five 2- $\mu$ m thick coronal sections backward from the forehead pole were made using a tissue slicer, incubated in TCC staining solution (Sigma-Aldrich Corporation, St. Louis, MO, USA) at $37^{\circ} \mathrm{C}$ for $30 \mathrm{~min}$, then fixed in fresh $10 \%$ formaldehyde for at least $24 \mathrm{~h}$. Following TCC staining, normal brain tissue is red while infarction tissue is white. The successful model was characterized by white cerebral infarction lesions located in the cortex and striatum and overlapped by the MCA dominant region.

\section{Preparation of histological slices}

At indicated time points, the rats were ip anesthetized with $10 \%$ chloral hydrate and positioned on the dissection table. The left ventricle was intubated after the thoracotomy, washed with cool saline to remove blood, fixed overnight with cool $4 \%$ formaldehyde in phosphate buffer, further immersed in 20 and $30 \%$ sucrose in phosphate buffer successively, and stored at $4^{\circ} \mathrm{C}$ until required. Frozen consecutive coronal slices (10- $\mu \mathrm{m}$ thick) were taken from the temporal lobe, and take one of each four was used for conventional Nissl staining, bromodeoxyuridine (BrdU) $\mathrm{IHC}$, and for the negative control.

\section{IHC of BrdU}

BrdU staining was carried out using the streptavidin-biotin complex method (BrdU was provided by Wuhan Boster Biological Engineering Company, Wuhan, China). Briefly, slices were washed twice with $0.01 \mathrm{M}$ phosphate-buffered saline (PBS), $\mathrm{pH} 7.3$, for $5 \mathrm{~min}$, and immersed in $0.3 \% \mathrm{H}_{2} \mathrm{O}_{2}$ PBS solution for 15 min to deactivate endogenous peroxidase and enhance antibody detection sensitivity, and then normal calf serum (1:20 dilution) was added to the slices at $37^{\circ} \mathrm{C}$ for $30 \mathrm{~min}$; the excess liquid was discarded and primary mouse anti-BrdU monoclonal antibody (1:100 dilution, Wuhan Boster Biological Engineering Company) was added to the slices overnight at $4^{\circ} \mathrm{C}$ (with further incubation at $37^{\circ} \mathrm{C}$ for $1 \mathrm{~h}$ if necessary). Incubation with biotin-conjugated goat anti-mouse IgG (Wuhan Boster Biological Engineering Company) at $37^{\circ} \mathrm{C}$ for 30 min was followed by development with a 3,3'-diaminobenzidine- $\mathrm{H}_{2} \mathrm{O}_{2}$ kit (Wuhan Boster Biological Engineering Company) for 20-30 min. During each step, except removal of the calf serum and addition of the primary antibody, slices were washed three times with $0.01 \mathrm{M}$ PBS for $5 \mathrm{~min}$. Finally, the slices were 
dehydrated conventionally, made transparent, and sealed with coverslips. The negative control was prepared by following the same procedure except that the primary antibody was replaced with PBS.

\section{Imaging analysis}

A HPIAS-2000 imaging analysis system (OLMPUS company in Japan) was applied to analyze the images of the cortex adjacent to the infarctions at 200X magnification. For quantification of BrdU-positive cells in per field, each slide was quantified by 15 power fields in the same area.

\section{Statistical analysis}

The Student $t$-test and analysis of variance were performed to compare the difference in positive cell numbers in each group. $\mathrm{P}<0.01$ was considered to be significant.

\section{RESULTS}

BrdU-positive cells, characterized by deeply stained nuclei in round cells, were barely observed in the cerebral cortexes and occasionally in the granule cell layers of the cortex in the control and sham-operation groups. Seven days after model establishment, IHC revealed that BrdU-positive cells in the operation and MK-801 groups were round, oval, spindle-shaped, or triangular and of different sizes in irregular arrangements. In addition, BrdU-positive cells massed, cytoskeletal structure in the infarctions was destroyed, neurons swelled, intercellular spaces widened, and disruption and apoptosis of the neurons were observed. The differences in the number of BrdU-positive cells were not significant between pairs of adjacent groups: the operation group, the 0.2-mg/kg group, the 0.4-mg/kg group, and the 0.6- $\mathrm{mg} / \mathrm{kg}$ group $(P>0.01)$, which indicated that the change of MK-801 concentration between pairs of adjacent groups did not correlate with the degree of inhibition of positive cell proliferation. However, the $t$-test showed significant differences between pairs of nonadjacent groups $(P<0.01)$. The numbers of BrdU-positive cells in the 0.8-, 1.0-, and 1.2-mg/kg groups were statistically different from those in the operation, 0.2-, 0.4-, and 0.6-mg/kg groups $(P<0.01)$. Furthermore, the differences in the number of BrdU-positive cells were significant between pairs of adjacent groups: $0.8-, 1.0-$, and 1.2-mg/kg groups $(P<0.01$, Figure 1 and Table 1$)$.

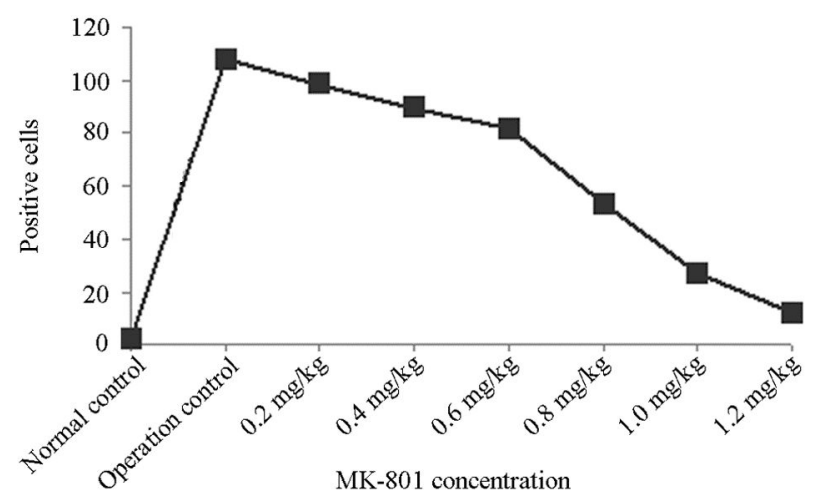

Figure 1. Variation trend of the number of BrdU positive cortical cells adjacent to the focus of infarct in MK-801 groups with different concentrations. 
Table 1. Counting and statistical results of the number of BrdU positive cortical cells adjacent to the focus of infarct in MK-801 groups with different concentrations (means $\pm S D, N=6$ ).

\begin{tabular}{ccccccccc}
\hline & Control & Operation & $0.2 \mathrm{mg} / \mathrm{kg}$ & $0.4 \mathrm{mg} / \mathrm{kg}$ & $0.6 \mathrm{mg} / \mathrm{kg}$ & $0.8 \mathrm{mg} / \mathrm{kg}$ & $1.0 \mathrm{mg} / \mathrm{kg}$ & $1.2 \mathrm{mg} / \mathrm{kg}$ \\
$(1)$ & $(2)$ & $(3)$ & $(4)$ & $(5)$ & $(6)$ & $(7)$ & $(8)$ \\
\hline Total number & $2.63 \pm 0.64$ & $107.43 \pm 16.29$ & $99.02 \pm 10.61$ & $89.97 \pm 9.86$ & $81.82 \pm 7.62$ & $53.53 \pm 4.64$ & $27.01 \pm 2.89$ & $12.29 \pm 1.02$ \\
\hline
\end{tabular}

1 , between (1) and other groups, $P<0.01 ; 2$, between (2) and (3), $P>0.01$, between (2) and other groups, $P<0.01$; 3 , between ( 3 ) and (4), $P>0.01$, between (3) and other groups, $P<0.01 ; 4$, between (4) and (5), $P>0.01$, between (4) and other groups, $P<0.01 ; 5$, between (5) and (6), $P>0.01$, between (5) and other groups, $P<0.01 ; 6$, between (6) and (7) (8), $P<0.01 ; 7$, between (7) and (8), $P<0.01$.

\section{DISCUSSION}

It has been widely accepted that the adult central nervous system does not have the ability to proliferate, differentiate, self-renew, and self-repair. However, further research on NSCs has shown that after adults suffer ischemia and hypoxia injury, some physical and chemical stimulation can promote nerve fiber cell proliferation and differentiation into neurons that can establish functional synaptic connections, and effectively restore impaired function (Nakatomi et al., 2002; Burns et al., 2009; Kernie and Parent, 2010). BrdU, a thymine analogue, can be incorporated into new DNA during DNA synthesis ( $\mathrm{S}$ phase), and BrdU antibody does not cross-react with thymine. Thus, IHC can be used to observe BrdU incorporation and cell proliferation (Cooper-Kuhn and Kuhn, 2002). BrdU-labeling is currently considered an indirect sign of NSCs. We used this principle and method to label proliferative cortical cells and explore the proliferation of cortical cells under normal and cerebral ischemia conditions; the results revealed that BrdU-positive cell proliferation markedly increased after cerebral ischemia in rats, which indicates that ischemic injury does induce cell proliferation in the germinal zone of the brain.

Takagi et al. (1999) established an adult rat model of transient global cerebral ischemia, labeled mitotic cells with BrdU, and observed subgranular zone NSC proliferation; the results revealed that the number of BrdU-positive cells was significantly increased 3, 7, 10, and 17 days after reperfusion, especially on Day 7 , which was consistent with the conclusions drawn by Yagita et al. (2001). Zhang et al. (2001) reported that NSCs proliferated after ischemic injury in the cerebral cortex ipsilateral to the injury and in the subventricular zone in MCAO adult rats, but only a few cells differentiated into astrocytes and the rest underwent apoptosis.

There are a number of hypotheses regarding the source of NSCs in the cortex adjacent to the infarction and the pathway that regulates NSC proliferation (Dizon et al., 2010; Toyoda et al., 2012; Kang et al., 2013). The subventricular and subgranular zones have been confirmed as the areas with largest numbers of NSCs in the adult brain. John et al. (1996) demonstrated that persistent perfusion of the lateral ventricle with epidermal growth factor in adult chinchillas increased the number of retrovirus-labeled cells by more than 17 times that of the control group perfused with normal saline, and simultaneously promoted the migration of newborn cells from the lateral ventricle wall to the membrane of adjacent regions (such as the cortex and the corpus striatum, etc.). This migration is called the rostral migratory stream and it functions through neural cell adhesion molecules. Other researchers have discovered that endogenous NSCs originally in the "dormant" state are activated after cerebral ischemia; cells proliferate and differentiate on both sides, ipsilateral and contralateral to ischemia, and mature cells surrounding the ischemic focus can revert to NSCs that re-proliferate and differentiate into newborn cells and migrate into the ischemic area where they eventually become thesocytes. This process is regulated by endogenous 
and exogenous cytokines, and is directly influenced by various signals of the internal environment (Zhang et al., 2001; Takasawa et al., 2002). In our study, the source of the BrdU-positive cells in the infarctions requires further confirmation.

Our research confirmed the presence of abundant BrdU-positive cells in the cortex adjacent to the infarctions. Through statistical analysis of positive cell numbers in the control, operation, and MK-801 groups, we conclude that: the number of BrdU-positive cells in the 0.4- and 0.6-mg/kg MK801 groups significantly decreased compared with the operation group; and the inhibitory effect of BrdU-positive cell proliferation at a MK-801 concentration of $0.8 \mathrm{mg} / \mathrm{kg}$ was markedly enhanced. We observed that the number of BrdU-positive cells declined with increasing MK-801 concentration, especially above $0.8 \mathrm{mg} / \mathrm{kg}$. This demonstrates the evident inhibitory effect on BrdU-positive cell proliferation of MK-801 at $0.4 \mathrm{mg} / \mathrm{kg}$; increasing MK-801 concentration (above $0.8 \mathrm{mg} / \mathrm{kg}$ ) positively correlated with this inhibitory effect. The mechanism of the protective effect of NMDA receptor antagonist MK-801 on neurons and its inhibitory effect on NSCs remain unclear. Most researchers believe that MK-801 promotes NSC proliferation through inhibition of EAA toxicity, simultaneously reduces calcium ion concentration, maintains the balance of intracellular and extracellular calcium, and suppresses cell apoptosis (Laeng et al., 2004; Langton et al., 2007; Kim et al., 2009). In addition, the mitogen of NSCs is basic fibroblast growth factor (bFGF), which, together with other cytokines, mainly regulates the division of early precursor cells and promotes NSC migration (Gleeson et al., 1999; Pearson et al., 2008). After cerebral ischemia, MK-801 regulates bFGF expression in the brain and promotes directional migration of NSCs sensitive to bFGF. Moreover, NMDA receptor antagonist can promote the upregulation of some cytokines such as IL-1 $\beta, I L-6$, and TNF- $\alpha$ after cerebral ischemia, thereby influencing the proliferation and differentiation of NSCs (Numakawa et al., 2010). However, the mechanisms and effects of MK-801 on neurons and NSCs remain unclear and require further investigation. In conclusion, MK-801 concentration correlates with the restoration of neural function after cerebral infarction; whether cortical NSCs adjacent to the infarctions can further differentiate into functional neurons to repair damaged neural networks requires further research.

\section{Conflicts of interest}

The authors declare no conflict of interest.

\section{REFERENCES}

Anderson DJ and Michelsohn A (1989). Role of glucocorticoids in the chromaffin-neuron developmental decision. Int. J. Dev. Neurosci. 7: 475-487.

Burns TC, Verfaillie CM and Low WC (2009). Stem cells for ischemic brain injury: a critical review. J. Comp. Neurol. 515: 125-144 Cooper-Kuhn CM and Kuhn HG (2002). Is it all DNA repair? Methodological considerations for detecting neurogenesis in the adult brain. Brain Res. Dev. Brain Res. 134: 13-21.

Dizon M, Szele F and Kessler JA (2010). Hypoxia-ischemia induces an endogenous reparative response by local neural progenitors in the postnatal mouse telencephalon. Dev. Neurosci. 32: 173-183.

Gage FH (2000). Mammalian neural stem cells. Science 287: 1433-1438.

Gleeson JG, Lin PT, Flanagan LA and Walsh CA (1999). Doublecortin is a microtubule-associated protein and is expressed widely by migrating neurons. Neuron 23: 257-271.

John KK, Hazel TG, Muller T, Dugich-Djordjevic MM, et al. (1996). Single factors direct the differentiation of stem cells from the fetal and adult central nervous system. Genes Dev. 10: 3129-3140.

Kang SS, Keasey MP, Arnold SA, Reid R, et al. (2013). Endogenous CNTF mediates stroke-induced adult CNS neurogenesis in mice. Neurobiol. Dis. 49: 68-78. 
Kernie SG and Parent JM (2010). Forebrain neurogenesis after focal Ischemic and traumatic brain injury. Neurobiol. Dis. 37: 267-274.

Kim Y, Comte I, Szabo G, Hockberger P, et al. (2009). Adult mouse subventricular zone stem and progenitor cells are sessile and epidermal growth factor receptor negatively regulates neuroblast migration. PLoS One 4: e8122.

Laeng P, Pitts RL, Lemire AL, Drabik CE, et al. (2004). The mood stabilizer valproic acid stimulates GABA neurogenesis from rat forebrain stem cells. J. Neurochem. 91: 238-251.

Langton JM, Kim JH, Nicholas J and Richardson R (2007). The effect of the NMDA receptor antagonist MK-801 on the acquisition and extinction of learned fear in the developing rat. Learn. Mem. 14: 665-668.

Longa EZ, Weinstein PR, Carlson S and Cummins R (1989). Reversible middle cerebral artery occlusion without craniectomy in rats. Stroke 20: 84-91.

Nakatomi H, Kuriu T, Okabe S, Yamamoto S, et al. (2002). Regeneration of hippocampal pyramidal neurons after ischemic brain injury by recruitment of endogenous neural progenitors. Cell 110: 429-441.

Numakawa T, Suzuki S, Kumamaru E, Adachi N, et al. (2010). BDNF function and intracellular signaling in neurons. Histol. Histopathol. 25: 237-258.

Pearson S, Sroczynska P, Lacaud G and Kouskoff V (2008). The stepwise specification of embryonic stem cells to hematopoietic fate is driven by sequential exposure to Bmp4, activin A, bFGF and VEGF. Development 135: 1525-1535.

Takagi Y, Nozaki K, Takahashi J, Yodoi J, et al. (1999). Proliferation of neuronal precursor cells in the dentate gyrus is accelerated after transient forebrain ischemia in mice. Brain Res. 831: 283-287.

Takasawa K, Kitagawa K, Yagita Y, Sasaki T, et al. (2002). Increased proliferation of neural progenitor cells but reduced survival of newborn cells in the contralateral hippocampus after focal cerebral ischemia in rats. J. Cereb. Blood Flow Metab. 22: 299-307.

Toyoda T, Matsukawa N, Sagisaka T, Uematsu N, et al. (2012). Suppression of astrocyte lineage in adult hippocampal progenitor cells expressing hippocampal cholinergic neurostimulating Peptide precursor in an in vivo ischemic model. Cell Transplant. 21: 2159-2169.

Yagita Y, Kitagawa K, Ohtsuki T, Takasawa K, et al. (2001). Neurogenesis by progenitor cells in the ischemic adult rat hippocampus. Stroke 32: 1890-1896.

Zhang RL, Zhang ZG, Zhang L, Chopp M (2001). Proliferation and differentiation of progenitor cells in the cortex and the subventricular zone in the adult rat after focal cerebral ischemia. Neuroscience 105: 33-41. 